\title{
\begin{tabular}{l|l} 
Mitraries & DSpace@MIT
\end{tabular}
}

MIT Open Access Articles

Superanalysis of the interference effect on adaptive antenna systems

The MIT Faculty has made this article openly available. Please share how this access benefits you. Your story matters.

Citation: Youngmin Jeong, Hyundong Shin, and M.Z. Win. "Superanalysis of the Interference Effect on Adaptive Antenna Systems." GLOBECOM 2010, 2010 IEEE Global Telecommunications Conference. 2010. 1-5. Copyright (C) 2010, IEEE

As Published: http://dx.doi.org/10.1109/GLOCOM.2010.5683404

Publisher: Institute of Electrical and Electronics Engineers

Persistent URL: http://hdl.handle.net/1721.1/65944

Version: Final published version: final published article, as it appeared in a journal, conference proceedings, or other formally published context

Terms of Use: Article is made available in accordance with the publisher's policy and may be subject to US copyright law. Please refer to the publisher's site for terms of use. 


\section{Superanalysis of the Interference Effect on Adaptive Antenna Systems}

\author{
Youngmin Jeong and Hyundong Shin \\ Department of Electronics and Radio Engineering \\ Kyung Hee University, Korea \\ Email: $\{$ yjeong,hshin\}@khu.ac.kr
}

\author{
Moe Z. Win \\ Laboratory for Information and Decision Systems \\ Massachusetts Institute of Technology, USA \\ Email: moewin@mit.edu
}

\begin{abstract}
We analyze the performance of optimum combining in a general cochannel interference environment with thermal noise. Specifically, we consider multiple unequal-power interferers, each is spatially correlated across receiving antennas. We develop a new mathematical methodology to analyze the average symbol error probability (SEP) of optimum combining diversity systems in Rayleigh fading. The analysis resorts to the so-called Berezin's supermathematics that treats both commuting and Grassmann anticommuting variables on an equal footing. This superanalysis framework enables us to derive the exact SEP expression for an arbitrary number of interferers with spatial correlation and possibly different power levels. Our results therefore encompass all the previous analytical results, based on the theory of multivariate statistics relating to complex Wishart matrices, for equal-power and/or spatially-uncorrelated interferers. Connecting the powerful supermathematical framework to the analysis of wireless diversity systems with optimum combining, we quantify the interference effects in terms of the degree of power unbalance and the amount of spatial correlation.
\end{abstract}

\section{INTRODUCTION}

In the presence of cochannel interference, adaptive antenna arrays with linear diversity combining have long been of interest to maximize the signal-to-interference-plus-noise ratio (SINR) [1]-[9]. The seminal work of [1], [2] laid the early foundations for optimum combining or minimum meansquare error (MMSE) diversity combining to improve the error performance and capacity of wireless communication systems by suppressing multiple interfering signals as well as by mitigating multipath fading of the desired signal. Subsequent to these early work, a large wave of publications has been spawned on the analysis of the average or outage error performance of optimum combining in detail including: i) the exact analysis [3]-[7]; ii) the interference-limited analysis whereby the thermal noise was ignored [8]; and iii) bounds and/or approximations [9]. Most of analyses were based on the joint eigenvalue distribution of complex Wishart matrices or the distributional theory of complex random matrices, for example: 1) equal-power spatially-uncorrelated interferers [3], [4], [8], [9]; 2) unequal-power spatially-uncorrelated interferers [5], [6]; and 3) equal-power spatially-correlated interferers [7]. ${ }^{1}$ However, there are no reported results for a more general

\footnotetext{
${ }^{1}$ Throughout the paper, we use for brevity the term "(un)equal-power spatially-(un)correlated interferers" to denote (un)equal-power interferers, each is spatially (un)correlated across receiving antennas.
}

cochannel interference environment taking into account an arbitrary number of interferers with both unequal power levels and spatial correlation. The main difficulty in the analysis arises due to the absence of a computationally tractable form of the joint eigenvalue distribution of complex random matrices relevant to the SINR in this general setting.

In this paper, we analyze the performance of optimum combining in a general cochannel interference environment with thermal noise. More specifically, we consider multiple unequal-power (UP) interferers, each is spatially correlated (SC) across receiving antennas. This general setting is referred to as a "UP-SC" interference environment. We develop a new mathematical methodology to analyze the average symbol error probability (SEP) of optimum combining diversity systems in such UP-SC interference environments. The key to the success of our approach relies on employing the Berezin's supermathematics [10]-[12]. The supermathematics treats the mathematical analysis and algebra of functions of both commuting and anticommuting variables on the same footing. Using this superanalysis methodology, we lay the foundation for deriving the exact SEP expression in the UPSC interference environments. Therefore, our results provide a unifying framework for analyzing diversity systems with optimum combining, covering all the previous exact SEP results (e.g., [3]-[6]) as special cases, and thereby we quantify the interference effects on the diversity performance (e.g., the average SEP) in terms of the degree of power unbalance and the amount of spatial correlation. Throughout the paper, we shall use the same notation as in [13] and also omit the detailed derivations due to a space constraint.

\section{PRELIMINARIES AND SyStem MODEL}

\section{A. Definitions}

Definition 1 ( $k$-Determinant): For a positive integer $m$ and $\mathscr{A} \subseteq\{1,2, \ldots, m\}$, let $\mathscr{C}_{m, k}^{(\mathscr{A})}$ be the set of all $k$-subsets of $\{1,2, \ldots, m\} \backslash \mathscr{A}:$

$$
\mathscr{C}_{m, k}^{(\mathscr{A})} \triangleq\{\mathscr{S}: \mathscr{S} \subseteq\{1,2, \ldots, m\} \backslash \mathscr{A},|\mathscr{S}|=k\} .
$$

For an $m \times m$ matrix $\boldsymbol{Q}$, the $k$-determinant of $\boldsymbol{Q}$ associated with the subset $\mathscr{A}$, denoted by $\operatorname{det}_{[k: \mathscr{A}]}(\boldsymbol{Q})$, is $\operatorname{defined}$ as the $k$ th elementary symmetric function of the eigenvalue subset 
of $Q$ :

$$
\operatorname{det}_{[k: \mathscr{A}]}(\boldsymbol{Q}) \triangleq \sum_{\mathscr{B} \in \mathscr{C}_{m, k}^{(\mathscr{A})}} \prod_{i \in \mathscr{B}} \lambda_{i}(\boldsymbol{Q})
$$

with the convention $\operatorname{det}_{[0: \mathscr{A}]}(\boldsymbol{Q})=1$.

Definition 2 (Complex Gaussian Matrix): An $m \times n$ complex random matrix $\boldsymbol{X}$ is said to have a $(p, q)$-rank complex Gaussian distribution if its entries are jointly complex Gaussian and the covariance of $\operatorname{vec}(\boldsymbol{X})$ has a Kronecker structure $\boldsymbol{\Psi}^{T} \otimes \boldsymbol{\Sigma}$, where $\boldsymbol{\Sigma}$ and $\boldsymbol{\Psi}$ are $m \times m$ and $n \times n$ positive semidefinite matrices of rank $p \leq m$ and $q \leq n$, respectively.

We use the notation $\boldsymbol{X} \sim \tilde{\mathcal{N}}_{m, n}^{p, q}(\boldsymbol{M}, \boldsymbol{\Sigma}, \boldsymbol{\Psi})$ to denote $\boldsymbol{X}$ is the $(p, q)$-rank complex Gaussian matrix with $\mathbb{E}\{\boldsymbol{X}\}=\boldsymbol{M}$. If $\boldsymbol{\Sigma}$ and $\boldsymbol{\Psi}$ are positive definite (i.e., $p=m$ and $q=n$ ), we simply denote $\boldsymbol{X} \sim \tilde{\mathcal{N}}_{m, n}(\boldsymbol{M}, \boldsymbol{\Sigma}, \boldsymbol{\Psi})$ as considered in [13].

Definition 3 (Detquotient Mean): Given $\xi>0$, the detquotient mean of the complex Gaussian matrix $X \sim$ $\tilde{\mathcal{N}}_{m, n}^{p, q}(\mathbf{0}, \boldsymbol{\Sigma}, \boldsymbol{\Psi})$, denoted by DQM $(\boldsymbol{\Sigma}, \boldsymbol{\Psi} ; \xi)$, is defined as

$$
\operatorname{DQM}(\boldsymbol{\Sigma}, \boldsymbol{\Psi} ; \xi) \triangleq \mathbb{E}_{\boldsymbol{X}}\left\{\frac{\operatorname{det}\left(\boldsymbol{I}_{m}+\boldsymbol{X} \boldsymbol{X}^{\dagger}\right)}{\operatorname{det}\left(\boldsymbol{I}_{m}+\xi \boldsymbol{X} \boldsymbol{X}^{\dagger}\right)}\right\} \text {. }
$$

\section{B. System Model}

We consider a wireless communication system with $N_{\mathrm{A}}$ antenna reception in the presence of $N_{\mathrm{I}}$ cochannel interferers with thermal noise in the UP-SC interference environment, where the fading rate is assumed to be much slower than the symbol rate. After matched filtering and sampling at the symbol rate, the output signal vector is given by

$$
\boldsymbol{r}=\sqrt{P_{0}} \boldsymbol{\alpha}_{0} s_{0}+\sum_{k=1}^{N_{\mathrm{I}}} \sqrt{P_{k}} \boldsymbol{\alpha}_{k} s_{k}+\boldsymbol{n}
$$

where $P_{0}$ and $P_{k}$ are the average received powers; $\boldsymbol{\alpha}_{0} \sim$ $\tilde{\mathcal{N}}_{N_{\mathrm{A}}, 1}\left(\mathbf{0}, \boldsymbol{I}_{N_{\mathrm{A}}}, 1\right)$ and $\boldsymbol{\alpha}_{k} \sim \tilde{\mathcal{N}}_{N_{\mathrm{A}}, 1}^{\kappa_{1}, 1}(\mathbf{0}, \boldsymbol{\Phi}, 1)$ are the channel gains; $s_{0}$ and $s_{k}$ are the desired and interfering signals with $\mathbb{E}\left\{\left|s_{0}\right|^{2}\right\}=\mathbb{E}\left\{\left|s_{k}\right|^{2}\right\}=1$; and $\boldsymbol{n} \sim \tilde{\mathcal{N}}_{N_{\mathrm{A}}, 1}\left(\mathbf{0}, N_{0} \boldsymbol{I}_{N_{\mathrm{A}}}, 1\right)$ is the additive white Gaussian noise (AWGN) vector, each element has the one-sided power spectral density $N_{0} \cdot{ }^{2}$ Note that the noise $\boldsymbol{n}$ and channel vectors $\boldsymbol{\alpha}_{i}, i=0,1, \ldots, N_{\mathrm{I}}$, are mutually independent, and the covariance matrix $\Phi$ of rank $\kappa_{1}$ captures the spatial correlation structure of each interfering signal.

Let $\boldsymbol{z}=\sum_{k=1}^{N_{\mathrm{I}}} \sqrt{P_{k}} \boldsymbol{\alpha}_{k} s_{k}+\boldsymbol{n}$ be the total interference-plusnoise term and INR $\mathrm{IN}_{k}=P_{k} / N_{0}$ be the average interference-tonoise power ratio (INR) per branch corresponding to the $k$ th interferer. Conditioned on the $\boldsymbol{\alpha}_{i}$ 's, the interference-plus-noise covariance matrix can be written as

$$
\boldsymbol{R}=N_{0}\left(\boldsymbol{I}_{N_{\mathrm{A}}}+\boldsymbol{H}_{\mathrm{I}} \boldsymbol{H}_{\mathrm{I}}^{\dagger}\right)
$$

\footnotetext{
${ }^{2}$ The subscripts 0 and $k \in\left\{1,2, \ldots, N_{\mathrm{I}}\right\}$ stand for the desired user and $k$ th interferer, respectively.
}

where

$$
\boldsymbol{H}_{\mathrm{I}}=\left[\begin{array}{lll}
\sqrt{\mathrm{INR}_{1}} \boldsymbol{\alpha}_{1} & \cdots & \sqrt{\mathrm{INR}_{N_{\mathrm{I}}}} \boldsymbol{\alpha}_{N_{\mathrm{I}}}
\end{array}\right] \sim \tilde{\mathcal{N}}_{N_{\mathrm{A}}, N_{\mathrm{I}}}^{\kappa_{1}, \kappa_{2}}(\mathbf{0}, \boldsymbol{\Phi}, \boldsymbol{\Lambda})
$$

with the INR profile matrix $\boldsymbol{\Lambda}=\operatorname{diag}\left(\mathrm{INR}_{1}, \ldots, \mathrm{INR}_{N_{\mathrm{I}}}\right){ }^{3}$ Note that we can in general consider that any $N_{\mathrm{I}} \times N_{\mathrm{I}}$ matrix $\grave{\Lambda}$ such that $\lambda_{k}(\grave{\Lambda})=\mathrm{INR}_{k}$ is equivalent to the INR profile $\Lambda$. If some of interferers have a negligible power level, the INR profile $\Lambda$ is effectively rank deficient. Hence, the rank $\kappa_{2}$ of $\Lambda$ reflects the effective number of nonnegligible interferers. With the weighting vector $\boldsymbol{w}_{\mathrm{oc}}=c \boldsymbol{R}^{-1} \boldsymbol{\alpha}_{0}$ (where $c$ is an arbitrary constant), the maximum SINR at the optimum combiner output $\boldsymbol{w}_{\mathrm{oc}}^{\dagger} \boldsymbol{r}$ is [1]

$$
\gamma=\operatorname{SNR} \boldsymbol{\alpha}_{0}^{\dagger}\left(\boldsymbol{I}_{N_{\mathrm{A}}}+\boldsymbol{H}_{\mathrm{I}} \boldsymbol{H}_{\mathrm{I}}^{\dagger}\right)^{-1} \boldsymbol{\alpha}_{0}
$$

where SNR $=P_{0} / N_{0}$ denotes the average SNR per branch.

\section{Symbol ERror Probability}

For any arbitrary two-dimensional signaling constellation with polygonal decision boundaries, the average SEP for coherent detection can be written as [14]

$$
P_{\mathrm{e}}=\sum_{k=1}^{M} p_{k} \sum_{\ell \in \mathcal{B}_{k}} \frac{1}{2 \pi} \int_{0}^{\phi_{k, \ell}} \mathcal{M}_{\gamma}\left(\frac{w_{k, \ell}}{4 \sin ^{2}\left(\theta+\psi_{k, \ell}\right)}\right) d \theta
$$

where $M$ is the constellation size; $\left\{p_{k}\right\}_{k=1}^{M}$ are the a priori symbol probabilities; $\phi_{k, \ell}, w_{k, \ell}, \psi_{k, \ell}$, and $\mathcal{B}_{k}$ are the quantities depending on the geometry of the constellation (see [14, Fig. 10]); and $\mathcal{M}_{\gamma}(s) \triangleq \mathbb{E}\left\{e^{-s \gamma}\right\}$ is the moment generating function (MGF) of $\gamma$. Using (6), we get

$$
\mathcal{M}_{\gamma}(s)=(1+s \mathrm{SNR})^{-N_{\mathrm{A}}} \mathrm{DQM}\left(\boldsymbol{\Phi}, \boldsymbol{\Lambda} ; \frac{1}{1+s \mathrm{SNR}}\right) \text {. }
$$

\section{A. Detquotient Mean DQM $(\boldsymbol{\Sigma}, \Psi ; \xi)$}

Using the Berezin's supermathematics, we first present the analytical solution for $\operatorname{DQM}(\boldsymbol{\Sigma}, \boldsymbol{\Psi} ; \xi)$, which enables us to derive the exact SEP expression for optimum combining in the UP-SC interference environments.

Lemma 1: Let $Q \in \mathbb{C}^{m \times m}$ be positive semidefinite. Then, we have the following inverse Fourier transforms for determinantal functions of $Q$ :

i) For a positive constant $\zeta$,

$$
\begin{aligned}
& \mathcal{F}_{(\omega)}^{-1}\left\{\operatorname{det}\left(\boldsymbol{I}_{m}+\jmath \omega \zeta \boldsymbol{Q}\right)^{-1}\right\}(t) \\
& =\sum_{i=1}^{\varrho(\boldsymbol{Q})} \sum_{j=1}^{\tau_{i}(\boldsymbol{Q})} \frac{\mathcal{X}_{i, j}(\boldsymbol{Q}) t^{j-1}}{\Gamma(j) \zeta^{j} \lambda_{\langle i\rangle}^{j}(\boldsymbol{Q})} \exp \left(-\frac{t}{\zeta \lambda_{\langle i\rangle}(\boldsymbol{Q})}\right) u(t)
\end{aligned}
$$

where $\jmath=\sqrt{-1}, \Gamma(z)$ is the Euler's gamma function, $u(z)$ is the Heaviside step function, and $\mathcal{X}_{i, j}(\boldsymbol{Q})$ is the $(i, j)$ th characteristic coefficient of $\boldsymbol{Q}[13$, Definition 4$] .^{4}$

\footnotetext{
${ }^{3}$ Since the fading rate is much slower than the symbol rate, the interference-plus-noise vector $\boldsymbol{z}$ conditioned on the interference channel vectors $\boldsymbol{\alpha}_{1}, \boldsymbol{\alpha}_{2}, \ldots, \boldsymbol{\alpha}_{N_{\mathrm{I}}}$ is $\sim \tilde{\mathcal{N}}_{N_{\mathrm{A}}, 1}(\mathbf{0}, \boldsymbol{R}, 1)$.

${ }^{4}$ We shall denote the inverse Fourier transform of $X(\jmath \omega)$ by

$$
\mathcal{F}_{(\omega)}^{-1}\{X(\jmath \omega)\}(t)=\frac{1}{2 \pi} \int_{-\infty}^{\infty} X(\jmath \omega) e^{\jmath \omega t} d \omega .
$$
}


ii) For an arbitrary constant $\zeta$,

$$
\mathcal{F}_{(\omega)}^{-1}\left\{\operatorname{det}\left(\boldsymbol{I}_{m}+\jmath \omega \zeta \boldsymbol{Q}\right)\right\}(t)=\sum_{k=0}^{m} \zeta^{k} \operatorname{det}_{[k: \emptyset]}(\boldsymbol{Q}) \delta^{(k)}(t)
$$

where $\delta^{(k)}(z)$ denotes the $k$ th derivative of the Dirac's delta function $\delta(z)$.

Theorem 1: Let $\boldsymbol{\Sigma} \in \mathbb{C}^{m \times m}$ and $\boldsymbol{\Psi} \in \mathbb{C}^{n \times n}$ be positive semidefinite. Then, for $\xi>0$, we have

$$
\begin{aligned}
& \operatorname{DQM}(\boldsymbol{\Sigma}, \boldsymbol{\Psi} ; \xi)=\mathcal{G}(\boldsymbol{\Sigma}, \boldsymbol{\Psi}) \mathcal{J}(\boldsymbol{\Sigma}, \boldsymbol{\Psi} ; \xi) \\
& -\xi \sum_{k=1}^{m} \sum_{\ell=1}^{n}\left\{\lambda_{k}^{2}(\boldsymbol{\Sigma}) \lambda_{\ell}^{2}(\boldsymbol{\Psi}) \mathcal{H}_{k, \ell}(\boldsymbol{\Sigma}, \boldsymbol{\Psi})\right. \\
& \left.\quad \times \mathcal{J}\left(\left[\begin{array}{cc}
\boldsymbol{\Sigma} & 0 \\
0 & \lambda_{k}(\boldsymbol{\Sigma})
\end{array}\right],\left[\begin{array}{cc}
\boldsymbol{\Psi} & 0 \\
0 & \lambda_{\ell}(\boldsymbol{\Psi})
\end{array}\right] ; \xi\right)\right\}
\end{aligned}
$$

with

$$
\begin{aligned}
\mathcal{G}(\boldsymbol{\Sigma}, \boldsymbol{\Psi})= & \sum_{k=0}^{\min \{m, n\}} k ! \operatorname{det}_{[k: \emptyset]}(\boldsymbol{\Sigma}) \operatorname{det}_{[k: \emptyset]}(\boldsymbol{\Psi}) \\
\mathcal{H}_{p, q}(\boldsymbol{\Sigma}, \boldsymbol{\Psi})= & \sum_{k=0}^{\min \{m, n\}-1} k ! \operatorname{det}_{[k:\{p\}]}(\boldsymbol{\Sigma}) \operatorname{det}_{[k:\{q\}]}(\boldsymbol{\Psi}) \\
\mathcal{J}(\boldsymbol{\Sigma}, \boldsymbol{\Psi} ; \xi)= & \sum_{i=1}^{\varrho(\boldsymbol{\Sigma})} \sum_{j=1}^{\tau_{i}(\boldsymbol{\Sigma})} \sum_{p=1}^{\varrho(\boldsymbol{\Psi})} \sum_{q=1}^{\tau_{p}(\boldsymbol{\Psi})}\left\{\mathcal{X}_{i, j}(\boldsymbol{\Sigma}) \mathcal{X}_{p, q}(\boldsymbol{\Psi})\right. \\
& \left.\times{ }_{2} F_{0}\left(j, q ;-\xi \lambda_{\langle i\rangle}(\boldsymbol{\Sigma}) \lambda_{\langle p\rangle}(\boldsymbol{\Psi})\right)\right\}
\end{aligned}
$$

where ${ }_{p} F_{q}(\cdot)$ is the generalized hypergeometric function.

Corollary 1: For $\boldsymbol{\Sigma}=\sigma \boldsymbol{I}_{m}$ and $\boldsymbol{\Psi}=\rho \boldsymbol{I}_{n}$, we have

$$
\begin{aligned}
\operatorname{DQM} & \left(\sigma \boldsymbol{I}_{m}, \rho \boldsymbol{I}_{n} ; \xi\right)=T_{m, n}^{(\sigma, \rho)}{ }_{2} F_{0}(m, n ;-\xi \sigma \rho) \\
& -\xi \sigma^{2} \rho^{2} m n T_{m-1, n-12}^{(\sigma, \rho)} F_{0}(m+1, n+1 ;-\xi \sigma \rho)
\end{aligned}
$$

where

$$
T_{m, n}^{(\sigma, \rho)}=\sum_{k=0}^{\min \{m, n\}} k !(\sigma \rho)^{k}\left(\begin{array}{l}
m \\
k
\end{array}\right)\left(\begin{array}{l}
n \\
k
\end{array}\right)
$$

Corollary 2: For $\boldsymbol{\Sigma}=\sigma \mathbf{1}_{m}$ and $\boldsymbol{\Psi}=\rho \mathbf{1}_{n}$, we have

$$
\begin{array}{r}
\operatorname{DQM}\left(\sigma \mathbf{1}_{m}, \rho \mathbf{1}_{n} ; \xi\right)=(1+\sigma \rho m n){ }_{2} F_{0}(1,1 ;-\xi \sigma \rho m n) \\
-\xi(\sigma \rho m n)_{2}^{2} F_{0}(2,2 ;-\xi \sigma \rho m n) .
\end{array}
$$

\section{B. General Result}

Using (7), (8), and Theorem 1, we obtain the exact SEP expression for optimum combining in the UP-SC interference environment as (13), shown at the bottom of the page, where $\mathcal{P}_{k, \ell}(\boldsymbol{A}, \boldsymbol{B})$ for $m \times m$ and $n \times n$ positive-semidefinite matrices $A$ and $B$ is given by (14) with

$$
\begin{aligned}
\mathcal{V}_{i, j}^{\phi, \psi}(\nu, a, b) \triangleq \frac{1}{2 \pi} & \int_{0}^{\phi}\left(1+\frac{a}{4 \sin ^{2}(\theta+\psi)}\right)^{-\nu} \\
& \times{ }_{2} F_{0}\left(i, j ;-\frac{b}{1+\frac{a}{4 \sin ^{2}(\theta+\psi)}}\right) d \theta
\end{aligned}
$$

for $i, j, \nu \in \mathbb{N}$ and $a, b>0$. Note that the expression (13) is valid for arbitrary spatial correlation $\Phi$ and INR profile matrix $\Lambda$. Together with systematic implementations of the characteristic coefficients and $k$-determinant provide a simple and unifying way to calculate the exact SEP.

\section{Case Study}

As two extreme interference environments, we now particularize the average SEP in (13) for the best and worst interference environments when the total INR is fixed to

$$
\mathrm{INR}_{\mathrm{tot}} \triangleq \sum_{k=1}^{N_{\mathrm{I}}} \mathrm{INR}_{k}=N_{\mathrm{I}} \bar{\gamma}_{\mathrm{I}}
$$

1) Worst interference case: When the desired signal is disturbed by equal-power spatially-uncorrelated interfering signals, the optimum combining receiver suffers the most severe interference. For this worst case, i.e., $\boldsymbol{\Phi}=\boldsymbol{I}_{N_{\mathrm{A}}}$ and $\boldsymbol{\Lambda}=\bar{\gamma}_{\mathrm{I}} \boldsymbol{I}_{N_{\mathrm{I}}}$, we can reduce the average SEP (13) using Corollary 1 to

$$
\begin{aligned}
& P_{\mathrm{e}}^{\text {(worst) }}=\sum_{k=1}^{M} p_{k} \sum_{\ell \in \mathcal{B}_{k}}\left\{T_{N_{\mathrm{A}}, N_{\mathrm{I}}}^{(1, \mathrm{INR})} \mathcal{V}_{N_{\mathrm{A}}, N_{\mathrm{I}}}^{\phi_{k, \ell}, \psi_{k}, \ell}\left(N_{\mathrm{A}}, w_{k, \ell} \mathrm{SNR}, \bar{\gamma}_{\mathrm{I}}\right)\right. \\
& \left.-N_{\mathrm{A}} N_{\mathrm{I}} \bar{\gamma}_{\mathrm{I}}^{2} T_{N_{\mathrm{A}}-1, N_{\mathrm{I}}-1}^{(1, \mathrm{INR})} \mathcal{V}_{N_{\mathrm{A}}+1, N_{\mathrm{I}}+1}^{\phi_{k}, \psi_{k, \ell}}\left(N_{\mathrm{A}}+1, w_{k, \ell} \mathrm{SNR}, \bar{\gamma}_{\mathrm{I}}\right)\right\}
\end{aligned}
$$

which is simple to evaluate.

$$
P_{\mathrm{e}}=\sum_{k=1}^{M} p_{k} \sum_{\ell \in \mathcal{B}_{k}}\left\{\mathcal{G}(\boldsymbol{\Phi}, \boldsymbol{\Lambda}) \mathcal{P}_{k, \ell}(\boldsymbol{\Phi}, \boldsymbol{\Lambda})-\sum_{r=1}^{N_{\mathrm{A}}} \sum_{t=1}^{N_{\mathrm{I}}} \lambda_{r}^{2}(\boldsymbol{\Phi}) \operatorname{INR}_{t}^{2} \mathcal{H}_{r, t}(\boldsymbol{\Phi}, \boldsymbol{\Lambda}) \mathcal{P}_{k, \ell}\left(\left[\begin{array}{cc}
\mathbf{\Phi} & 0 \\
0 & \lambda_{r}(\boldsymbol{\Phi})
\end{array}\right],\left[\begin{array}{cc}
\boldsymbol{\Lambda} & 0 \\
0 & \mathrm{INR}_{t}
\end{array}\right]\right)\right\}
$$

$$
\mathcal{P}_{k, \ell}(\boldsymbol{A}, \boldsymbol{B})=\sum_{i=1}^{\varrho(\boldsymbol{A})} \sum_{j=1}^{\tau_{i}(\boldsymbol{A})} \sum_{p=1}^{\varrho(\boldsymbol{B})} \sum_{q=1}^{\tau_{p}(\boldsymbol{B})} \mathcal{X}_{i, j}(\boldsymbol{A}) \mathcal{X}_{p, q}(\boldsymbol{B}) \mathcal{V}_{j, q}^{\phi_{k, \ell}, \psi_{k, \ell}}\left(m, w_{k, \ell} \mathrm{SNR}, \lambda_{\langle i\rangle}(\boldsymbol{A}) \lambda_{\langle p\rangle}(\boldsymbol{B})\right)
$$


2) Best interference case: Let $\boldsymbol{\Lambda}=\bar{\gamma}_{\mathrm{I}} \mathbf{1}_{N_{\mathrm{I}}}$ for the circumstance in the presence of a dominant strong-power interferer, which is equivalent to the case of INR profile $\left\{\mathrm{INR}_{k}\right\}_{k=1}^{N_{\mathrm{I}}}=$ $\left\{N_{\mathrm{I}} \bar{\gamma}_{\mathrm{I}}, 0, \ldots, 0\right\}$. In this degenerate interference-power situation together with spatially full correlation $\Phi=1_{N_{\mathrm{A}}}$, we can reduce the average SEP (13) using Corollary 2 to

$$
\begin{aligned}
& P_{\mathrm{e}}^{\text {(best) }}=\sum_{k=1}^{M} p_{k} \sum_{\ell \in \mathcal{B}_{k}}\left\{\left(1+N_{\mathrm{A}} N_{\mathrm{I}} \bar{\gamma}_{\mathrm{I}}\right)\right. \\
& \quad \times \mathcal{V}_{1,1}^{\phi_{k, \ell}, \psi_{k, \ell}}\left(N_{\mathrm{A}}, w_{k, \ell} \mathrm{SNR}, N_{\mathrm{A}} N_{\mathrm{I}} \bar{\gamma}_{\mathrm{I}}\right) \\
& \left.\quad-\left(N_{\mathrm{A}} N_{\mathrm{I}} \bar{\gamma}_{\mathrm{I}}\right)^{2} \mathcal{V}_{2,2}^{\phi_{k, \ell}, \psi_{k, \ell}}\left(N_{\mathrm{A}}+1, w_{k, \ell} \mathrm{SNR}, N_{\mathrm{A}} N_{\mathrm{I}} \bar{\gamma}_{\mathrm{I}}\right)\right\} .
\end{aligned}
$$

\section{Achievable Diversity}

Theorem 2: Let $\mathrm{SIR}_{k}=P_{0} / P_{k}, k=1,2, \ldots, N_{\mathrm{I}}$, be the average signal-to-interference power ratio (SIR) for the $k$ th interferer and

$$
d_{\mathrm{oc}} \triangleq \lim _{\substack{\mathrm{SNR} \rightarrow \infty \\ \mathrm{SIR} \mathrm{R}_{k} \rightarrow c_{k}}} \frac{-\log P_{\mathrm{e}}}{\log \mathrm{SNR}}
$$

be the diversity order achieved by optimum combining where $c_{k}$ 's are finite positive constants. Then, the diversity order is

$$
d_{\mathrm{oc}}=N_{\mathrm{A}}-\min \left\{\kappa_{1}, \kappa_{2}\right\} .
$$

Theorem 2 reveals that the rank-deficient interference correlation or INR profile decreases the effective number of interferers and hence only $\min \left\{\kappa_{1}, \kappa_{2}\right\}$ receiving antennas are required to suppress the interfering signals. Therefore, regardless of the number of interferers and their power profile, if each interfering signal is fully correlated at the receiver, one of receiving antennas can be exploited to suppress these degenerate interfering signals and the other antennas can realize the diversity order of $N_{\mathrm{A}}-1$.

\section{NumericAl Results}

Fig. 1 shows the analytical and simulated average SEP results for 8-PSK versus SNR when $N_{\mathrm{I}}=3$ and $N_{\mathrm{A}}$ varies from 2 to 5 . In this example, the spatial correlation for interfering signals follows the exponential correlation $\boldsymbol{\Phi}=\left[0.5^{|i-j|}\right]$ and the SIR's for three interferers are set to $\left\{\mathrm{SIR}_{k}\right\}_{k=1}^{N_{\mathrm{I}}}=\{0 \mathrm{~dB}, 5 \mathrm{~dB}, 10 \mathrm{~dB}\}$. We can see that the analysis agrees exactly with the simulation results and observe that the achievable diversity order (i.e., the high-SNR slope of the SEP curve) is equal to $d_{\mathrm{oc}}=N_{\mathrm{A}}-\min \left\{N_{\mathrm{A}}, N_{\mathrm{I}}\right\}$, as stated in Theorem 2, since $\boldsymbol{\Phi}$ and $\boldsymbol{\Lambda}$ are full rank. Hence, the high-SNR slope vanishes for overloaded cases $\left(N_{\mathrm{A}} \leq N_{\mathrm{I}}\right)$ in which all antennas are used up to suppress the interfering signals.

The effect of interference correlation on the SEP performance can be ascertained by referring to Fig. 2 where the analytical SEP of 8-PSK at SNR $=25 \mathrm{~dB}$ is depicted as a function of the correlation coefficient $\rho$ for the exponential interference correlation $\boldsymbol{\Phi}=\left[\rho^{|i-j|}\right]$. In this figure, $N_{\mathrm{I}}, N_{\mathrm{A}}$, and $\left\{\mathrm{SIR}_{k}\right\}_{k=1}^{N_{\mathrm{I}}}$ are the same as in Fig. 1. We can observe

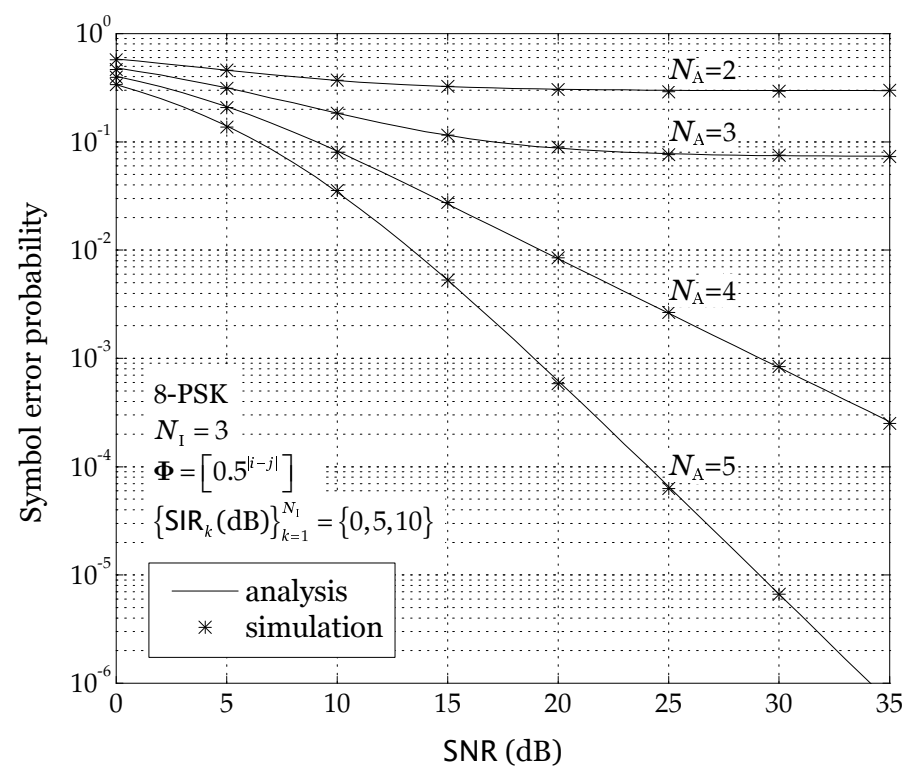

Fig. 1. Average SEP of 8-PSK versus SNR: $N_{\mathrm{I}}=3, \boldsymbol{\Phi}=\left[0.5^{|i-j|}\right]$, SIR $\left\{\mathrm{SIR}_{k}\right\}_{k=1}^{N_{\mathrm{I}}}=\{0 \mathrm{~dB}, 5 \mathrm{~dB}, 10 \mathrm{~dB}\}$, and $N_{\mathrm{A}}=2,3,4,5$.

that the SEP decreases with the correlation coefficient $\rho$, i.e., the amount of correlation. This SEP decrease is especially pronounced, due to strong interference correlation, at the value of $\rho$ close to one. This reveals that the SEP as a functional of the eigenvalues of the interference correlation matrix $\Phi$ is monotonically decreasing in a sense of Schur (MDS), namely, the more spatially-correlated interference results in the less severe interfering effect, and hence less significantly degrades the SEP performance. ${ }^{5}$

In what follows, we consider an intraclass INR profile matrix for interfering signals:

$$
\boldsymbol{\Lambda}_{\text {intra }}(\rho)=\bar{\gamma}_{\mathrm{I}}\left[\begin{array}{ccccc}
1 & \rho & \rho & \cdots & \rho \\
\rho & 1 & \rho & \cdots & \rho \\
\vdots & \vdots & \vdots & \ddots & \vdots \\
\rho & \rho & \rho & \cdots & 1
\end{array}\right]_{N_{\mathrm{I}} \times N_{\mathrm{I}}} \quad, \quad \rho \in[0,1]
$$

which is equivalent to the INR profile

$$
\begin{aligned}
\left\{\operatorname{INR}_{k}\right\}_{k=1}^{N_{\mathrm{I}}} & =\left\{\lambda_{k}\left(\boldsymbol{\Lambda}_{\text {intra }}(\rho)\right)\right\}_{k=1}^{N_{\mathrm{I}}} \\
& =\left\{\left(1-\rho+N_{\mathrm{I}} \rho\right) \bar{\gamma}_{\mathrm{I}},(1-\rho) \bar{\gamma}_{\mathrm{I}}, \ldots,(1-\rho) \bar{\gamma}_{\mathrm{I}}\right\} .
\end{aligned}
$$

Note that the intraclass INR profile can be interpreted as a situation consisting of one strong interferer and $N_{\mathrm{I}}-1$ relatively low equal-power interferers with the aggregate INR equal to $I N R_{\text {tot }}=N_{\mathrm{I}} \bar{\gamma}_{\mathrm{I}}$.

Fig. 3 demonstrates the effect of interference-power profile on the SEP performance, where the analytical SEP for 8-PSK versus SNR is depicted for the intraclass INR profile $\boldsymbol{\Lambda}_{\text {intra }}(\rho)$. In this example, $N_{\mathrm{A}}=4, N_{\mathrm{I}}=3, \boldsymbol{\Phi}=\left[0.5^{|i-j|}\right], \bar{\gamma}_{\mathrm{I}}=\mathrm{SNR}$, and $\rho=0$ (equal-power interferers), $0.3,0.5,0.7,0.8,0.9$,

\footnotetext{
${ }^{5}$ See, e.g., [13, Appendix I] and references therein for the basic concept of Schur monotonicity.
} 


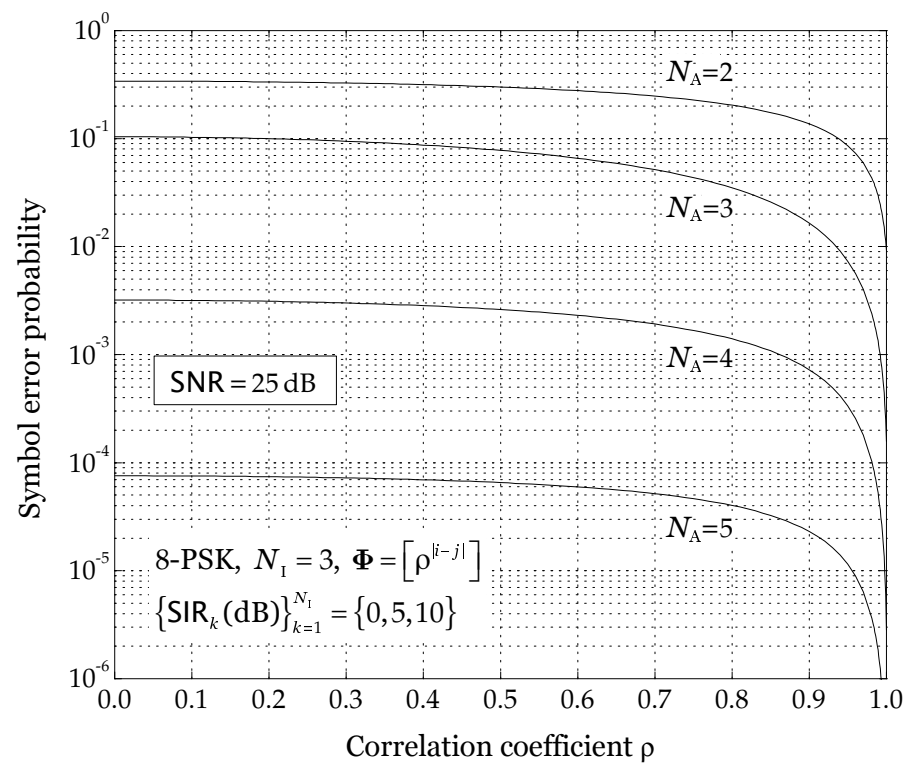

Fig. 2. Average SEP of 8-PSK as a function of the correlation coefficient $\rho$ at $\mathrm{SNR}=25 \mathrm{~dB}: N_{\mathrm{I}}=3, \boldsymbol{\Phi}=\left[\rho^{|i-j|}\right], \operatorname{SIR}\left\{\mathrm{SIR}_{k}\right\}_{k=1}^{N_{\mathrm{I}}}=$ $\{0 \mathrm{~dB}, 5 \mathrm{~dB}, 10 \mathrm{~dB}\}$, and $N_{\mathrm{A}}=2,3,4,5$.

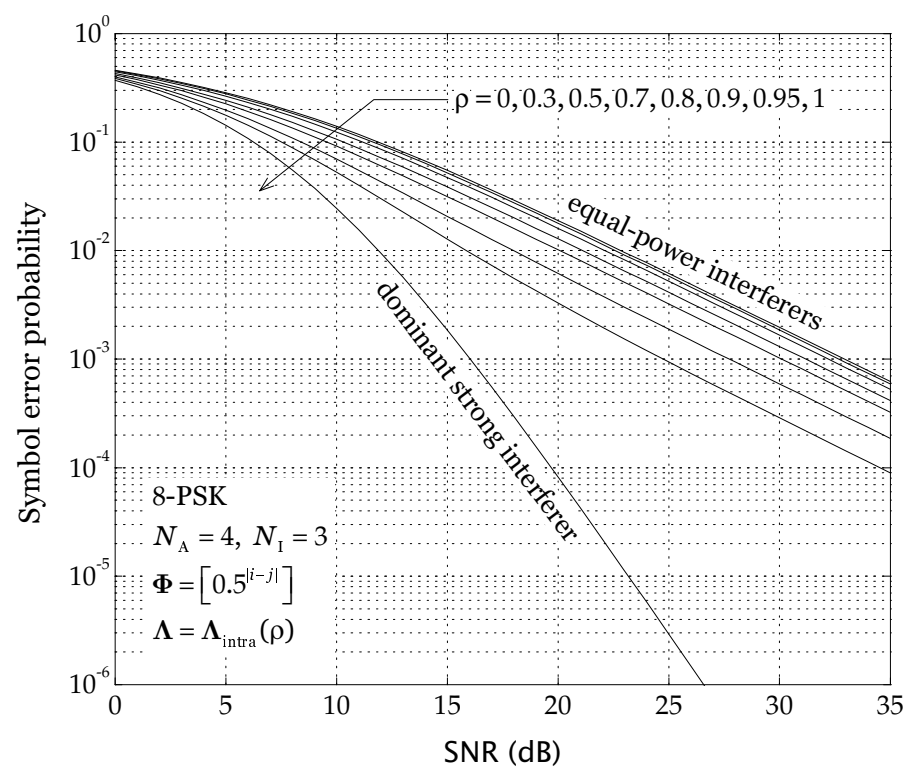

Fig. 3. Average SEP of 8-PSK versus SNR: $N_{\mathrm{A}}=4, N_{\mathrm{I}}=3, \boldsymbol{\Phi}=$ $\left[0.5^{|i-j|}\right], \boldsymbol{\Lambda}=\boldsymbol{\Lambda}_{\text {intra }}(\rho), \bar{\gamma}_{\mathrm{I}}=\mathrm{SNR}$, and $\rho=0$ (equal-power interferers), $0.3,0.5,0.7,0.8,0.9,0.95,1$ (dominant strong interferer).

0.95, 1 (dominant strong interferer). We can observe that the SEP decreases with $\rho$, i.e., the degree of power unbalance among interferers. In particular, the achievable diversity order is equal to $d_{\mathrm{oc}}=3$ (i.e., $d_{\mathrm{oc}}=4-\min \{3,1\}$ ) for $\rho=1$ (dominant strong interferer) due to the rank deficiency of $\boldsymbol{\Lambda}$, while achieving the diversity order equal to $d_{\mathrm{oc}}=1$ (i.e., $\left.d_{\mathrm{oc}}=4-\min \{3,3\}\right)$ for other values of $\rho$. This reveals that the SEP as a functional of the INR profile $\left\{\mathrm{INR}_{k}\right\}_{k=1}^{N_{\mathrm{I}}}$ (or equivalently, the eigenvalues of the INR profile matrix $\boldsymbol{\Lambda}$ ) is again MDS, namely, the more unbalanced (or less spread out) interference-power profile results in the less severe interfering effect, providing a better SEP performance.

\section{CONCLUSiOnS}

We derived the exact average SEP for optimum combining in a flat Rayleigh-fading environment in the presence of multiple cochannel interferers and thermal noise, taking into account unequal power levels among interfering signals, each is spatially correlated across receiving antennas. Using the Berezin's supermathematics, we established a unifying framework to calculate the exact SEP for such general interference environments. On account of the generality of interference modeling, our results are substantial enough to encompass all the previous exact SEP analysis for equal-power and/or spatially-uncorrelated interferers as special cases, which hence consolidate the effects of interference power unbalance and spatial correlation on the SEP of diversity systems.

\section{ACKNOWLEDGMENT}

This work was supported by the National Research Foundation of Korea (NRF) grant funded by the Korea government (MEST) (No. 2010-0014773).

\section{REFERENCES}

[1] J. H. Winters, "Optimum combining in digital mobile radio with cochannel interference," IEEE J. Sel. Areas Commun., vol. 2, no. 4, pp. 528-539, Jul. 1984.

[2] — , "Optimum combining for indoor radio systems with multiple users," IEEE Trans. Commun., vol. 35, no. 11, pp. 1222-1230, Nov. 1987.

[3] M. Chiani, M. Z. Win, and A. Zanella, "Error probability for optimum combining of $M$-ary PSK signals in the presence of interference and noise," IEEE Trans. Commun., vol. 51, no. 11, pp. 1949-1957, Nov. 2003.

[4] D. Lao and A. M. Haimovich, "Exact closed-form performance analysis of optimum combining with multiple cochannel interferers and Rayleigh fading," IEEE Trans. Commun., vol. 51, no. 6, pp. 995-1003, Jun. 2003.

[5] M. Chiani, M. Z. Win, and A. Zanella, "On optimum combining of $M$-PSK signals with unequal-power interferers and noise," IEEE Trans. Commun., vol. 53, no. 1, pp. 44-47, Jan. 2005.

[6] P. J. Smith, "Exact performance analysis of optimum combining with multiple interferers in flat Rayleigh fading," IEEE Trans. Commun., vol. 55, no. 9, pp. 1674-1677, Sep. 2007.

[7] D. Yue, X. Wang, and F. Xu, "Performance analysis for optimum combining of Rayleigh fading signals with correlated Rayleigh interferers and noise," IEEE Signal Process. Lett., vol. 13, no. 5, pp. 269-272, May 2006.

[8] M. Kang, M.-S. Alouini, and L. Yang, "Outage probability and spectrum efficiency of cellular mobile radio systems with smart antennas," IEEE Trans. Commun., vol. 50, no. 12, pp. 1871-1877, Dec. 2002.

[9] M. Chiani, M. Z. Win, A. Zanella, R. K. Mallik, and J. H. Winters, "Bounds and approximations for optimum combining of signals in the presence of multiple cochannel interferers and thermal noise," IEEE Trans. Commun., vol. 51, no. 2, pp. 296-307, Feb. 2003.

[10] F. A. Berezin, The Method of Second Quantization. New York: Academic Press, 1966.

[11] — Introduction to Superanalysis, ser. Mathematical Physics and Applied Mathematics, A. A. Kirillov, Ed. New York: Springer, 1987, vol. 9.

[12] K. Efetov, Supersymmetry in Disorder and Chaos. Cambridge, United Kingdom: Cambridge University Press, 1997.

[13] H. Shin and M. Z. Win, "MIMO diversity in the presence of double scattering," IEEE Trans. Inf. Theory, vol. 54, no. 7, pp. 2976-2996, Jul. 2008.

[14] M. Z. Win, P. C. Pinto, and L. A. Shepp, "A mathematical theory of network interference and its applications," Proc. IEEE, vol. 97, no. 2, pp. 205-230, Feb. 2009. 